\title{
The Joint Estimation of Term Structures and Credit Spreads ${ }^{1}$
}

\author{
Patrick Houweling ${ }^{2}$ \\ Jaap Hoek $^{3}$ \\ Frank Kleibergen ${ }^{4}$
}

March 23, 1999

\footnotetext{
${ }^{1}$ Part of the research was conducted while the first author was affiliated with the Quantitative Research department of the Robeco Group. The authors thank Ton Vorst for many valuable suggestions and critical remarks, Rabobank International for providing the data, Michel van der Spek for preparing the data sets and Kees van den Berg and Peter Ferket for useful comments on an earlier version of the paper.

${ }^{2}$ Central Market Risk, Rabobank International: P.O. Box 17100, 3500 HG Utrecht, The Netherlands, +31-302166566 and Tinbergen Institute, Erasmus University Rotterdam: P.O. Box 1738, 3000 DR Rotterdam, The Netherlands, +31-10-4088938, houweling@few.eur.nl.

${ }^{3}$ Quantitative Research, Robeco Group: P.O. Box 973, 3000 AZ Rotterdam, The Netherlands, +31-10-2242799, j.hoek@robeco.nl.

${ }^{4}$ Econometric Institute, Erasmus University Rotterdam: P.O. Box 1738, 3000 DR Rotterdam, The Netherlands, +31-10-4088938, kleibergen@few.eur.nl.
} 


\begin{abstract}
We present a new framework for the joint estimation of the default-free government term structure and corporate credit spread curves. By using a high-quality data set of German mark denominated bonds, we show that this yields more realistic spreads than conventionally obtained spread curves that result from subtracting independently estimated government and corporate term structures. The estimated spread curves are now smooth functions of time to maturity, as opposed to the twisting curves one gets from the traditional method, and are less sensitive to model specifications. Moreover, the implied corporate term structures have tighter confidence intervals.
\end{abstract}




\section{Introduction}

The growth of the corporate bond market, the rapid development of the market for credit derivatives and the growing awareness among investors of credit risk in derivative products, has led to a surge in the development of new models. For the successful implementation of pricing models for corporate bonds or credit derivatives and of models that asses derivatives' credit risk, an accurate description of the term structures of interest rates for different credit risk classes is a prerequisite. Measuring a term structure for a particular debtor or rating class boils down to calculating its credit spread curve relative to the government curve, which is assumed to be default-free. Traditionally, spread curves are calculated by subtracting independently estimated government and corporate term structures. In this paper we present a new framework for term structure estimation that jointly estimates the government curve and credit spread curves. Unlike the twisting curves one gets from the traditional method, the estimated spread curves are now smooth functions of time to maturity, and are less sensitive to model settings. Moreover, the implied corporate term structures have tighter confidence intervals, i.e. they are more reliably estimated.

The joint estimation of term structures is based on the decomposition of a risky term structure into a default-free curve and a credit spread curve. Since the default-free curve is taken from the government curve, our model for a corporate term structure can focus on the credit spread only and can thus be parsimonious. All model parameters, i.e. from both the government curve and the corporate spread curve, are jointly estimated from a combined data set of bonds. Compared to the traditional approach, a smaller number of parameters is estimated from a larger data set. The model is applied to a data set of German mark denominated bonds, whose credit ratings range from Standard and Poor's ratings AAA to B, and obtain smooth and reliably estimated spread curves that are relatively robust to model misspecification. We demonstrate that these results can be attributed to both the joint and the parsimonious modelling. Independently estimating the government curve and a parsimoniously specified corporate curve model does not yield the same results, and neither does jointly estimating the government curve and a richly specified corporate spread curve.

The remainder of the paper is structured as follows. Section II presents the new framework for the joint estimation of the government term structure and corporate credit spread curves. The construction of confidence intervals for estimated term structures and spread curves is explained in section III . The model settings and characteristics of our data set are described in section IV . Section V applies the new model to this data set and compares the performance of jointly estimated term structures to independently estimated 
curves. Finally, section VI concludes the paper.

\section{Multi-Curve Model}

A corporate term structure is obtained by adding a term structure of credit spreads to the default-free government curve. Theoretical models of corporate bond prices, such as the models by Merton (1974), Longstaff and Schwartz (1995) and Jarrow, Lando and Turnbull (1997), imply smooth credit spread curves. For investment grade firms these models predict that the spread curve is a monotonically increasing function of time to maturity, while for speculative grade bonds a humped-shaped or downward sloping spread curve is expected. Recent empirical research by Helwege and Turner (1999) supported the hypothesis of increasing credit spreads for high-grade issuers, but also found statistically significant evidence of increasing spreads for lower rating categories.

Ideally, we would like to use a different spread curve for each firm, reflecting the uniqueness of a firm's characteristics that determine its credit risk. However, due to data constraints, which are discussed in section IV , we have to resort to grouping firms that have similar credit worthiness and face similar operating environments. We do this by categorising firms by credit rating and industry into $C$ categories, where category 1 corresponds to government bonds. The purpose is to estimate a spread curve for each category. One way of achieving this goal is by applying a standard term structure estimation procedure for each of the categories, see e.g. Anderson, Breedon, Deacon, Derry and Murphy (1996, chapter 2) for an extensive overview of term structure estimation methods. A separate model is specified for each category $c$ and the model parameters are estimated from a data set of bonds of that category. The credit spread curve is then calculated as the difference between the independently estimated term structures of categories $c$ and 1 (the government). In section $\mathrm{V}$, we illustrate that these calculations are likely to result in twisty spread curves that alternately have positively and negatively sloped segments. This contradicts the smooth curves that are predicted by the theoretical bond price models mentioned above. Moreover, the level and shape of the spread are shown to be sensitive to model misspecification.

Instead of independently estimating term structures, we propose a joint estimation approach. Since a corporate term structure consists of a default-free curve and a credit spread curve, it seems natural to only model the spread and take the default-free part from the government curve. Several representations of the 
term structure exist, such as by means of discount factors or spot interest rates, but it is common practice to model the discount curve. We therefore propose the following framework for jointly estimating the discount curves $^{1}$

$$
\begin{aligned}
& D_{1}(t)=d(t) \\
& D_{c}(t)=d(t)+s_{c}(t), c=2,3, \ldots, C,
\end{aligned}
$$

where $D_{c}(\cdot)$ is the discount curve of category $c, d(\cdot)$ is the model for the government discount curve and $s_{c}(\cdot)$ is the model for the discount spread curve of category $c$ with respect to the government curve. All parameters in the models for the government curve and the discount spread curves are jointly estimated from a combined data set of government and corporate bonds. We refer to this model as the multi-curve model as opposed to a single-curve model that independently estimates a single corporate term structure.

To model $d(\cdot)$ and $s_{c}(\cdot)$, we use spline functions, as introduced to the term structure estimation literature by McCulloch (1971). Some commonly used spline models are the exponential splines, by Vasicek and Fong (1982), and the $B$-splines, as discussed by Shea (1985) and Steeley (1991). Splines are tailored to approximate a scatter of data points by a continuous and preferably smooth function. Their main advantage is their flexibility: there is no need to a priori impose a particular curvature, because the shape of the curve is determined by the data. Bliss (1997) compared several non-parametric term structure estimation models and found that spline models perform at least as good as competing models, and outperform the other considered models if the data contains longer maturity bonds (over 5 years).

Splines are basically piecewise polynomials. The approximation interval ${ }^{2}[a, b]$ is divided into $n$ subintervals $\left[\tau_{0}, \tau_{1}\right],\left[\tau_{1}, \tau_{2}\right], \ldots,\left[\tau_{n-1}, \tau_{n}\right]$, where the knots $\tau_{i}$ are chosen such that $a=\tau_{0}<\tau_{1}<\ldots<\tau_{n}=b$. The data points in each subinterval are modelled as a $k^{\text {th }}$ degree polynomial. The $n$ polynomials are constrained by the condition that the spline has to be $k-1$ times continuously differentiable. This is a restriction at the knots

\footnotetext{
${ }^{1}$ Alternatively, we could model

$$
D_{c}(t)=m_{c}(t) d(t), c=2,3, \ldots, C,
$$

which contains a multiplicative spread $m_{c}(t)$ instead of an additive spread $s_{c}(t)$. The spot curve that corresponds to such a discount curve reads

$$
r_{c}(t)=\frac{-\ln D_{c}(t)}{t}=\frac{-\ln d(t)}{t}+\frac{-\ln m_{c}(t)}{t} \equiv r_{1}(t)+s_{c}^{*}(t)
$$

i.e. an additive spread on the government spot curve. Although this specification may be intuitively more appealing than an additive spread on the government discount curve, it is more difficult to estimate due to the nonlinearity of $D_{c}(t)$. Therefore, we leave this multiplicative model for a follow-up paper and focus here on the additive model.

${ }^{2}$ With term structure estimation the approximation interval runs from 0 to the longest bond maturity in the sample.
} 
$\tau_{1}, \tau_{2}, \ldots, \tau_{n-1}$ only and poses $k$ constraints on the coefficients of two adjacent polynomials. In sum, we have $n(k+1)$ coefficients minus $(n-1) k$ constraints, leaving only $n+k$ degrees of freedom. A more parsimonious way of representing splines is by means of basis functions, see e.g. Powell (1981, p. 228). Any $k^{\text {th }}$ degree spline function $S(\cdot)$ with knots $\boldsymbol{\tau}$ can be expressed as a linear combination of $n+k$ basis functions $\mathbf{f}(\cdot)=$ $\left\{f_{1}(\cdot), f_{2}(\cdot), \ldots, f_{n+k}(\cdot)\right\}$

$$
S(t)=\sum_{s=1}^{n+k} \alpha_{s} f_{s}(t)=\mathbf{f}(t)^{\prime} \boldsymbol{\alpha}
$$

Once the basis is chosen and the degree $k$ and the knots $\tau$ are set, the basis functions are fully specified. The spline weights $\boldsymbol{\alpha}$ however are unknown and will be estimated from the data. Powell (1981) recommends the use of a basis of $B$-spline functions, because of their efficiency and numerical stability. Steeley (1991) details the use of $B$-splines for term structure estimation.

We use $B$-splines to model the government discount curve and corporate discount spread curves in (1). We set $d(t)=\mathbf{g}_{1}(t)^{\prime} \boldsymbol{\beta}_{1}$ and $s_{c}(t)=\mathbf{g}_{c}(t)^{\prime} \boldsymbol{\beta}_{c}$, where $\mathbf{g}_{i}(\cdot)$ contains $n_{i}+k_{i} B$-spline basis functions that span a spline of $k_{i}^{t h}$ degree with knots $\boldsymbol{\tau}_{i}$; section IV discusses the specification of the degrees and knots. The multi-curve model (1) is then rewritten as

$$
\begin{aligned}
& D_{1}(t)=\mathbf{g}_{1}(t)^{\prime} \boldsymbol{\beta}_{1} \\
& D_{c}(t)=\mathbf{g}_{1}(t)^{\prime} \boldsymbol{\beta}_{1}+\mathbf{g}_{c}(t)^{\prime} \boldsymbol{\beta}_{c}, c=2,3, \ldots, C .
\end{aligned}
$$

To estimate the unknown spline weights $\boldsymbol{\beta}_{1}, \boldsymbol{\beta}_{2}, \ldots, \boldsymbol{\beta}_{C}$, we construct a data set, consisting of $B_{c}$ bonds of category $c$, and use the discounted cash flow (DCF) equation to link the bond prices to a discount curve. The DCF equation expresses the theoretical price of the $b^{\text {th }}$ bond of category $c$ as the sum of discounted cash flows

$$
P_{D C F, c b}=\sum_{i=1}^{N_{c b}} C F_{c b i} D_{c}\left(t_{c b i}\right)
$$

where $P_{D C F, c b}$ is the theoretical bond price, $N_{c b}$ is the number of cash flows and $C F_{c b i}$ is the $i^{\text {th }}$ cash flow that is paid at time $t_{c b i}$. By using the DCF equation as the bond price model, we have to confine the data set to bonds whose characteristics are in line with its assumptions. This amounts to including fixed-income bonds with known redemptions and excluding any bonds with optional elements - such as callable and puttable 
bonds - and bonds with floating or index-linked coupons.

For category 1, i.e. for government bonds, we substitute (2a) into (3), yielding

$$
\begin{aligned}
P_{D C F, 1 b} & =\sum_{i=1}^{N_{1 b}} C F_{1 b i}\left(\sum_{s=1}^{n_{1}+k_{1}} \beta_{1 s} g_{1 s}\left(t_{1 b i}\right)\right)=\sum_{s=1}^{n_{1}+k_{1}} \beta_{1 s}\left(\sum_{i=1}^{N_{1 b}} C F_{1 b i} g_{1 s}\left(t_{1 b i}\right)\right) \\
& \equiv \sum_{s=1}^{n_{1}+k_{1}} x_{1 b s} \beta_{1 s}=\mathbf{x}_{1 b}^{\prime} \boldsymbol{\beta}_{1},
\end{aligned}
$$

whereas for categories $2,3, \ldots, C$ substitution of (2b) into (3) results in

$$
\begin{aligned}
P_{D C F, c b} & =\sum_{s=1}^{n_{1}+k_{1}} \beta_{1 s}\left(\sum_{i=1}^{N_{c b}} C F_{c b i} g_{1 s}\left(t_{c b i}\right)\right)+\sum_{s=1}^{n_{c}+k_{c}} \beta_{c s}\left(\sum_{i=1}^{N_{c b}} C F_{c b i} g_{c s}\left(t_{c b i}\right)\right) \\
& \equiv \sum_{s=1}^{n_{1}+k_{1}} x_{c b s} \beta_{1 s}+\sum_{s=1}^{n_{c}+k_{c}} y_{c b s} \beta_{c s}=\mathbf{x}_{c b}^{\prime} \boldsymbol{\beta}_{1}+\mathbf{y}_{c b}^{\prime} \boldsymbol{\beta}_{c}
\end{aligned}
$$

Note that these equations for the theoretical bond price are linear in the unknown parameters, because all terms in $\mathbf{x}$ and $\mathbf{y}$ are either known from the characteristics of the bond or the specification of the spline models. In order to estimate the spline weights, we substitute the theoretical prices $P_{D C F, c b}$ by observed market prices $P_{c b}$ and add an error term $\varepsilon_{c b}$ to the equations. The error term is necessary, because the DCF method is not able to perfectly explain bond prices. ${ }^{3}$ Using matrix notation, we obtain the following linear regression model

$$
\left[\begin{array}{c}
\mathbf{P}_{1} \\
\mathbf{P}_{2} \\
\mathbf{P}_{3} \\
\vdots \\
\mathbf{P}_{C}
\end{array}\right]=\left[\begin{array}{ccccc}
\mathbf{X}_{1} & \mathbf{0} & \mathbf{0} & \ldots & \mathbf{0} \\
\mathbf{X}_{2} & \mathbf{Y}_{2} & \mathbf{0} & \ldots & \mathbf{0} \\
\mathbf{X}_{3} & \mathbf{0} & \mathbf{Y}_{3} & \ldots & \mathbf{0} \\
\vdots & \vdots & \vdots & \ddots & \vdots \\
\mathbf{X}_{C} & \mathbf{0} & \mathbf{0} & \ldots & \mathbf{Y}_{C}
\end{array}\right]\left[\begin{array}{c}
\boldsymbol{\beta}_{1} \\
\boldsymbol{\beta}_{2} \\
\boldsymbol{\beta}_{3} \\
\vdots \\
\boldsymbol{\beta}_{C}
\end{array}\right]+\left[\begin{array}{c}
\varepsilon_{1} \\
\varepsilon_{2} \\
\varepsilon_{3} \\
\vdots \\
\varepsilon_{C}
\end{array}\right], \varepsilon_{c} \sim \text { i.i.d. }\left(0, \sigma_{c}^{2}\right)
$$

where $\mathbf{X}_{c}$ is a $\left(B_{c} \times\left(n_{1}+k_{1}\right)\right)$ matrix with rows $\left\{\mathbf{x}_{c 1}^{\prime}, \mathbf{x}_{c 2}^{\prime}, \ldots, \mathbf{x}_{c B_{c}}^{\prime}\right\}$ and $\mathbf{Y}_{c}$ is a $\left(B_{c} \times\left(n_{c}+k_{c}\right)\right)$ matrix with rows $\left\{\mathbf{y}_{c 1}^{\prime}, \mathbf{y}_{c 2}^{\prime}, \ldots, \mathbf{y}_{c B_{c}}^{\prime}\right\}$. We allow the disturbances to have different variances for each category, because prices of lower rated bonds are generally more noisy due to lower liquidity and a higher uncertainty about their perceived credit worthiness. ${ }^{4}$

\footnotetext{
${ }^{3}$ Of course it is possible to obtain an arbitrary high goodness of fit by increasing the number of parameters. However, the resulting term structure is likely to have a twisting shape and a wide confidence interval.

${ }^{4}$ Also, the residuals of independently estimated single-curve models can be shown to have significantly different variances using a heteroscedasticity test.
} 
Estimates $\hat{\boldsymbol{\beta}}_{1}, \hat{\boldsymbol{\beta}}_{2}, \ldots, \hat{\boldsymbol{\beta}}_{C}$ of the spline weights are readily obtained by applying Feasible Generalised Least Squares (FGLS) estimation to (5). ${ }^{5}$ Since we modelled the discount curves as continuous functions of time to maturity, we can evaluate $\hat{D}_{c}(t)$ for any maturity $t$. It is important to emphasise however, that splines are an interpolation technique. Therefore, discount factors for maturities beyond the maximum maturity bond become unreliable.

\section{Confidence Intervals}

Bond prices are not perfectly explained by our model. Consequently, the term structure cannot be measured exactly and values derived from it are subject to random measurement error. To evaluate the accuracy of estimated discount, spot, discount spread and spot spread curves, we construct confidence intervals for these estimates. For their construction, variances of the estimates are required, whose computation is detailed below.

With spline estimation it is straightforward to calculate the variance of an estimated discount factor for a specific maturity $t$. Since the estimate $\hat{D}_{c}(t)$ of the discount factor for maturity $t$ is a linear combination of the parameter estimates, we can apply the result that the variance of a linear combination $\mathbf{a}^{\prime} \boldsymbol{\xi}$ of a vector of random variables $\boldsymbol{\xi}$ with covariance matrix $\operatorname{cov}(\boldsymbol{\xi})$ is given by the quadratic form

$$
\operatorname{var}\left(\mathbf{a}^{\prime} \boldsymbol{\xi}\right)=\mathbf{a}^{\prime} \operatorname{cov}(\boldsymbol{\xi}) \mathbf{a}
$$

Hence,

$$
\begin{aligned}
& \operatorname{var}\left(\hat{D}_{1}(t)\right)=\mathbf{g}_{1}(t)^{\prime} \operatorname{cov}\left(\hat{\boldsymbol{\beta}}_{1}\right) \mathbf{g}_{1}(t), \text { and } \\
& \operatorname{var}\left(\hat{D}_{c}(t)\right)=\left(\mathbf{g}_{1}(t), \mathbf{g}_{c}(t)\right)^{\prime} \operatorname{cov}\left(\hat{\boldsymbol{\beta}}_{1}, \hat{\boldsymbol{\beta}}_{c}\right)\left(\mathbf{g}_{1}(t), \mathbf{g}_{c}(t)\right), c=2,3, \ldots, C .
\end{aligned}
$$

Similarly, the variance of an estimated discount spread $\hat{s}_{c}(t)=\hat{D}_{c}(t)-\hat{D}_{1}(t)$ equals

$$
\operatorname{var}\left(\hat{s}_{c}(t)\right)=\mathbf{g}_{c}(t)^{\prime} \operatorname{cov}\left(\hat{\boldsymbol{\beta}}_{c}\right) \mathbf{g}_{c}(t), c=2,3, \ldots, C
$$

It is also interesting to have an expression for the accuracy of the corresponding spot curves and spot spread

\footnotetext{
${ }^{5}$ Actually, we are using Restricted FGLS, because we impose the constraints that the discount functions have value 1 for maturity 0 , i.e. $D_{c}(0)=1$. This is a linear restriction on the spline weights, see $(2 \mathrm{a})$ and $(2 \mathrm{~b})$.
} 
curves. Because these curves depend non-linearly on the estimated parameters, their variances are not straightforwardly computed. A useful approximation is given by the following result, which is a generalisation of (6): the variance of a function $\phi(\cdot)$ of $\boldsymbol{\xi}$ is approximated by the quadratic form

$$
\operatorname{var}(\phi(\xi)) \approx\left(\frac{\partial \phi(\boldsymbol{\xi})}{\partial \boldsymbol{\xi}}\right)^{\prime} \operatorname{cov}(\boldsymbol{\xi})\left(\frac{\partial \phi(\xi)}{\partial \boldsymbol{\xi}}\right)
$$

The estimated spot rate $\hat{r}_{c}(t)$ is a function of the estimated discount factor $\hat{D}_{c}(t)$

$$
\hat{r}_{c}(t)=\phi\left(\hat{D}_{c}(t)\right)=-\frac{\ln \hat{D}_{c}(t)}{t},
$$

so that its variance is approximately

$$
\operatorname{var}\left(\hat{r}_{c}(t)\right) \approx\left(\frac{-1}{t \hat{D}_{c}(t)}\right) \operatorname{var}\left(\hat{D}_{c}(t)\right)\left(\frac{-1}{t \hat{D}_{c}(t)}\right)=\frac{\operatorname{var}\left(\hat{D}_{c}(t)\right)}{\left(t \hat{D}_{c}(t)\right)^{2}}
$$

To construct the variance for an estimated spot spread curve

$$
\hat{s}_{r, c}(t)=\hat{r}_{c}(t)-\hat{r}_{1}(t)=-\frac{\ln \left(\hat{d}(t)+\hat{s}_{c}(t)\right)}{t}+\frac{\ln \hat{d}(t)}{t}
$$

we write it explicitly as a function of the estimated parameters $\hat{\boldsymbol{\beta}}_{1}$ and $\hat{\boldsymbol{\beta}}_{c}$

$$
\hat{s}_{r, c}(t)=\phi\left(\hat{\boldsymbol{\beta}}_{1}, \hat{\boldsymbol{\beta}}_{c}\right)=-\frac{\ln \left(\mathbf{g}_{1}(t)^{\prime} \hat{\boldsymbol{\beta}}_{1}+\mathbf{g}_{c}(t)^{\prime} \hat{\boldsymbol{\beta}}_{c}\right)}{t}+\frac{\ln \left(\mathbf{g}_{1}(t)^{\prime} \hat{\boldsymbol{\beta}}_{1}\right)}{t} .
$$

Using (7), the variance is then approximated as

$$
\operatorname{var}\left(\hat{s}_{r, c}(t)\right) \approx\left(\frac{\partial \hat{s}_{r, c}(t)}{\partial \hat{\boldsymbol{\beta}}_{1}}, \frac{\partial \hat{s}_{r, c}(t)}{\partial \hat{\boldsymbol{\beta}}_{c}}\right)^{\prime} \operatorname{cov}\left(\hat{\boldsymbol{\beta}}_{1}, \hat{\boldsymbol{\beta}}_{c}\right)\left(\frac{\partial \hat{s}_{r, c}(t)}{\partial \hat{\boldsymbol{\beta}}_{1}}, \frac{\partial \hat{s}_{r, c}(t)}{\partial \hat{\boldsymbol{\beta}}_{c}}\right)
$$

where

$$
\begin{aligned}
\frac{\partial \hat{s}_{r, c}(t)}{\partial \hat{\boldsymbol{\beta}}_{1}} & =\frac{-\mathbf{g}_{1}(t)}{t\left(\mathbf{g}_{1}(t)^{\prime} \hat{\boldsymbol{\beta}}_{1}+\mathbf{g}_{c}(t)^{\prime} \hat{\boldsymbol{\beta}}_{c}\right)}+\frac{\mathbf{g}_{1}(t)}{t \mathbf{g}_{1}(t)^{\prime} \hat{\boldsymbol{\beta}}_{1}} \text { and } \\
\frac{\partial \hat{s}_{r, c}(t)}{\partial \hat{\boldsymbol{\beta}}_{c}} & =\frac{-\mathbf{g}_{c}(t)}{t\left(\mathbf{g}_{1}(t)^{\prime} \hat{\boldsymbol{\beta}}_{1}+\mathbf{g}_{c}(t)^{\prime} \hat{\boldsymbol{\beta}}_{c}\right)} .
\end{aligned}
$$




\section{Model Settings and Data}

\section{A Model Settings}

Before we are able to estimate the regression model (5), we have to specify the exact form of the basis functions. The functional form of the basis functions follows by choosing the degree of the splines and the number and location of the knots. These choices reflect the familiar trade-off between flexibility and smoothness. The degree of the splines should not be chosen too high, to preclude the problems of higher order polynomials ${ }^{6}$ If the order is too low however, the estimated curve will not fit the data very well. If the number of knots is chosen too low, the model will not be able to fit term structures with difficult shapes. On the other hand, if it is too high, the estimated curve is sensitive to outliers.

For the spline model for the government discount curve, we can use results from the term structure estimation literature. Almost all studies that employ spline functions to model the discount curve use third degree splines. ${ }^{7}$ Beim (1992) conducted a simulation study and concluded that cubic splines are preferable. Poirier (1976, p. 49) demonstrated that fitting a cubic spline minimises the integral of the square of the second derivative, which is an approximation of a function's smoothness, see also Adams and van Deventer (1994). Consequently, cubic splines are a convenient compromise between high goodness of fit and smooth curves.

With regard to the specification of the knots, there is less agreement. McCulloch (1971) proposed to set the number of knots equal to the integer nearest to the square root of the number of bonds in the sample. The knots are then located such that an approximately equal number of bonds is placed in each segment. Litzenberger and Rolfo (1984) stated that the McCulloch scheme is likely to result in a poor fit for longer maturities due to the larger number of shorter maturity bonds. As an alternative, they suggested to exogoneously place the knots at 1, 5 and 10 years, roughly corresponding to an economic segmentation into short, medium and long maturities. Langetieg and Smoot (1989) tested the McCulloch knot placement scheme against the economic scheme and found that the latter typically performed better. Steeley (1991) experimented with the specification of the knots and recommended placing knots at 5 and 10 years as a starting point for future research. The simulation study by Beim (1992) revealed that cubic splines with two knots minimised the standard error of

\footnotetext{
${ }^{6}$ Using higher order polynomials often results in spurious curvature between the data points, see e.g. Shea (1984, p.255). This is especially true if such polynomials are fitted to data that are not uniformly distributed over the approximation interval, as is the case with term structure estimation, see figure 1 in section $\mathrm{B}$.

${ }^{7}$ Only McCulloch (1971) used quadratic splines in his pioneering study, resulting in 'knuckles' in the forward curve. In his follow-up paper, McCulloch (1975) switched to cubic splines.
} 
fit between the estimated and the simulated 'true' discount curves.

We also use spline functions to model discount spread curves, but there is no prior evidence available on the specification of the degree and the knots. We provide a heuristic justification of the model settings in section V . Our choices are guided by the observation that a spread curve generally has a less complicated shape than a term structure. Therefore, we reduce the flexibility of the spline model for the spread curve by reducing the degrees of freedom. Compared to the spline for the discount curve of category $c$ in a single-curve model, we specify the discount spread curve $s_{c}(\cdot)$ in the multi-curve model as a lower degree spline with a smaller number of knots. That is, we use a quadratic spline function and the knots are chosen to be a subset of the knots of the single-curve spline model.

\section{B Data}

To appraise the performance of the proposed multi-curve model and compare it to independently estimated single-curve models, we use a data set of German Mark (DEM) denominated bonds. Their characteristics, like the maturity date, coupon percentage and rating, are obtained from Bloomberg, whereas bond quotes are retrieved daily at $4.00 \mathrm{pm}$ from Reuters' TREASURY and EUROBOND pages. ${ }^{8}$. For illustration, we present the results for the trading days of June 1998, yielding a total of 1291 quoted bonds. To estimate the term structures, we construct a sample of fixed-coupon, bullet bonds and to ensure their liquidity, we only use bonds that are quoted on at least 18 days of the 20 trading days of June 1998. There are 620 bonds that satisfy these restrictions.

In estimating the term structure on a particular trading day, we also consistently exclude all bonds with a maturity less than 1 year, because such bonds typically have low liquidity ${ }^{9}$. Instead, we add four synthetic zero-coupon bonds to the sample, whose prices correspond to 1-, 3- and 6-month and 1-year money-market rates, respectively (see also Bühler, Uhrig-Homburg, Walter and Weber, 1999, p. 15). We use Frankfurt Interbank Offered Rates (FIBORs) as money-market rates, but since most commercial banks have an AA rating these rates are not straightly applicable to other rating classes. Therefore, we correct the FIBORs by adding or subtracting a rating-dependent spread. The price $P_{c t}$ of a synthetic bond for category $c$ of maturity

\footnotetext{
${ }^{8}$ These Reuters pages are connected to broker pages, and each time a broker updates a quote for a bond, that quote is also refreshed on the Reuters page. Therefore, the TREASURY and EUROBOND pages provide a good representation of the market.

${ }^{9}$ There are at least three reasons for the illiquidity of bonds with a remaining maturity of less than 1 year. First, they are usually kept in portfolio until redemption to receive the principal. Second, due to their small duration, their price is relatively insensitive to changes in interest rates. Third, transaction costs influence short term yields more than longterm yields. These factors make short maturity bonds unpopular among investors.
} 
$t$ is thus computed as

$$
P_{c t}=\frac{1}{\left(1+\mathrm{FIBOR}_{t}+\operatorname{correction}_{c}\right)^{t}} .
$$

The number of suitable bonds of a single corporate debtor is limited. Three arguments explain this small sample size. First, a non-government debtor has smaller borrowing requirements, so that the number of issued bonds is typically rather small. Also, their amount outstanding is much smaller, which usually implies less liquidity. Second, for financial risk management purposes, many companies issue their bonds in different countries to offset currency exposures in those countries. As a result, the number of bonds denominated in a single currency can be rather small. Finally, many corporate bonds have embedded options and thus do not comply with the assumptions of the DCF model.

Therefore, we resort to grouping firms by rating and industry. First we show a division of the included bonds by rating, see table 1 . We use compounded, aggregated ratings, i.e. composed of Moody's and Standard \& Poor's major ratings, and consider AAA-rated government bonds as a separate category, indicated by 'rating' symbol GOVT. It is clear that the number of bonds per rating decreases with credit quality. Therefore, the reliable estimation of term structures of lower rating categories is hampered by an insufficient number of bonds if we were to use a single-curve method. The proposed multi-curve model offers a solution, because it only focuses on the spread curve and involves less parameters.

\section{[insert table 1 around here]}

The distribution of the bond's maturity dates is shown in figure 1. For government bonds and AAA bonds, large gaps in the maturity distribution are observed beyond a maturity of 10 years. This is caused by the very infrequent issuance of long term bonds (e.g. 30-year bonds), as opposed to the regular issuance of 5-year and 10-year bonds. Therefore, a term structure estimation procedure has few data points beyond 10 years and identification of that part of the term structure is more difficult. The confidence interval is therefore wider, i.e. the estimated curve is less reliable, in sparse maturity segments.

\section{[insert figure 1 around here]}

For credit classes AAA and AA there are enough bonds to allow a further classification by industry, but for the other ratings this is not feasible due to limited number of bonds. The included bonds from ratings 
AAA and AA are assigned to one of four compounded Reuters industry classifications. According to table 2, the majority of the bonds is issued by financial companies, whereas supra-national organisations are also well represented.

\section{[insert table 2 around here]}

\section{Results}

\section{A Single-Curve Results}

Before we discuss the results of the multi-curve (MC) model, we first present some estimation results of single-curve (SC) models. We only describe the results for the rating classes in table 1. The results for the industry classifications of table 1 are similar and do not provide additional insights. Table 3 summarises the specifications of the SC model. We use third degree $B$-splines for all bond categories, but the number and placement of the knot points differ. For the ratings GOVT, AAA, AA and A, we set the knots at 3 and 9 years, approximately corresponding to a segmentation into short-, medium- and long-term maturities. For the lower rating classes, $\mathrm{BBB}, \mathrm{BB}$ and $\mathrm{B}$, we use only one knot at 5 years, because the number of bonds in these classes is relatively small. The table also mentions the corrections to be applied to the AA-rated Frankfurt Interbank Offered Rates (FIBORs) in the calculation of the synthetic bonds' prices to take credit risk into account. ${ }^{10}$

\section{[insert table 3 around here]}

Table 4 summarises the term structure estimations for all 20 trading days of June 1998 by averaging three kinds of evaluation statistics: goodness of fit, smoothness and reliability. We measure goodness of fit as the Root Mean Squared Error (RMSE) of the residuals, calculated as the market price of a bond minus its theoretical DCF price (4a) or (4b). Following Poirier (1976, p. 49), we calculate the smoothness of estimated spot curves and spot spread curves as the integral of the square of their second derivative. The reliability of an estimated spot curve is indicated by its standard error, evaluated at maturities 2,5 and 10 years. Figure 2 graphically illustrates the SC estimation results for the first day of our data set, June $2^{\text {nd }}$, 1998, by depicting spot interest rate curves with their $95 \%$ confidence intervals, spot spread curves, residuals and standard errors

\footnotetext{
${ }^{10}$ The corrections are taken from Bloomberg and are inevitably approximations. Fortunately, the estimated curves are rather insensitive to small changes in these corrections.
} 
for the estimated discount curves. Note that we estimate discount curves and discount spread curves and that the corresponding spot curves and spot spread curves are subsequently calculated from these estimates.

\section{[insert table 4 around here] \\ [insert figure 2 around here]}

The residual scatter plots in figure $2 \mathrm{~b}$ show that the market prices of government bonds are reasonably approximated by the theoretical DCF prices, since all absolute deviations, except one, are less than DEM 0.25. The scatters for the bond categories AAA, AA, A and $\mathrm{BBB}$ are more dispersed, but the absolute errors are still smaller than DEM 1. Fitted prices for BB- and B-rated bonds are the least accurate with the largest residuals being about DEM 10. Similar conclusions are drawn from the RMSE statistic in table 4, which has the lowest value for GOVT bonds and generally increases for lower rated bonds. Apparently, the bonds in lower rating categories are increasingly more heterogeneous, which can be attributed to relative differences in perceived default probabilities and recovery rates of the issuers. Liquidity differences between the bonds can also contribute to the larger dispersion of pricing errors.

The extent to which market prices can be fitted accurately, has consequences for the reliability of the estimated curves. For the GOVT, AAA, AA and A curves, the confidence bounds in figure 2a almost coincide with the spot curve, whereas for the $\mathrm{BBB}, \mathrm{BB}$ and $\mathrm{B}$ curves the upper and lower bounds are distinctly observable in the graphs. Therefore, the reliability decreases with credit worthiness. The plotted standard errors in figure 2c and the reliability statistics in table 4 also show that the estimation error increases with maturity. This largely corresponds to the remark in section IV that confidence intervals are wide in maturity segments that contain a small number of bonds.

Most interesting for our purposes are the credit spread curves that can be obtained by subtracting the estimated corporate spot curve from the estimated government spot curve. All spot spread curves of June $2^{\text {nd }}$, 1998 in figure 2a have an unrealistically twisting shape, because they have alternately positively and negatively sloped segments. Especially the spread curves for investment-grade bonds display twisty behaviour. This can be explained from the relatively small magnitude of these spreads compared to interest rate levels: spot rates for classes AAA, AA, A and BBB are approximately $3.5 \%$ to $5.5 \%$, whereas spot spreads range from $0.1 \%$ to $0.6 \%$. Therefore, small errors in either the government or the corporate spot curve can result in substantial errors in the spread; in other words, any dissimilarity in the curves' curvatures implies twists in the spread curve. These fluctuations are indeed observed in the graphs. The twisting spread curves are not in line with 
the smooth spread curves that are predicted by the theoretical bond price models of Merton (1974), Longstaff and Schwartz (1995) and Jarrow et al. (1997). Also, the hypothesised positive slope for investment-grade bonds is violated.

\section{B Multi-Curve Results}

The MC model is expected to solve the abovementioned problems of SC models. We explicitly and parsimoniously model the spread curve, so that we are able to obtain smooth spread curves. Since the model for a corporate bond category now only has to focus on the discount spread relative to the default-free government discount curve, together with the use of a combined data set of government and corporate bonds, the parameters can be estimated more reliably. ${ }^{11}$ Therefore, the reliability of the estimated corporate curves improves.

Table 3 shows the specification of the degree and knots for the MC model. For the government discount curve, we use exactly the same settings as in the SC model, but for all corporate discount spread curves we lowered the degree and for some curves also the number of knots. Rating categories AAA, AA and A use quadratic splines with one knot at 9 years, while the BBB, BB and B discount spread curves are modelled as quadratic splines with one knot at 5 years. Later we show how these specifications were found, but first we show that they indeed yield the hypothesised favourable properties of the MC model over its SC competitor. Figure 3 provides a graphical representation of the results of June $2^{\text {nd }}, 1998$ and table 4 again summarises the main characteristics by averaging the goodness of fit, smoothness and reliability statistics over all 20 trading days of June 1998.

\section{[insert figure 3 around here]}

The graphs in figure 3a contain the estimated spot spread curves from the MC model and, for comparison, also the SC spot spreads from figure 2a. For all bond categories, there is a major improvement in the smoothness of the spread curves. Compared to the fluctuating SC spread curves, the MC spread curves are smooth, mostly increasing functions of time to maturity. However, most spread curves, except for ratings AA and A, are decreasing for the longest maturities. ${ }^{12}$ Table 4 adds quantitative evidence to these graphical

\footnotetext{
${ }^{11}$ Since the parameters of the model for the government discount curve are jointly estimated with the parameters of the corporate spread model, the estimated government curve differs from an independently estimated government curve. However, the changes are rather small and here we are only interested in the corporate curves.

${ }^{12}$ Helwege and Turner (1999) showed that this behaviour is attributable to a particular type of heterogeneity within a rating
} 
insights: the smoothness statistic is decimated for all rating categories, except B. The improvements range from a factor 5 for category A to 40 for BBB-rated bonds. Corporate spot curves, calculated as the sum of the estimated government and spread curves, also have become smoother, again with category B as the only exception. Note that the smoothness statistics for the spot spread curves are now much smaller than for the spot curves, confirming our prior belief in section IV that spreads have a less complicated shape.

The second advantage of the MC model over independently estimated SC models resides in the increased reliability of the estimated corporate term structures. ${ }^{13}$ Figure $3 \mathrm{c}$ and table 4 show that for all ratings and all maturities the standard errors of MC curves are lower than those of $\mathrm{SC}$ curves. The reliability for the $\mathrm{BB}$ and B categories improves most. For segments with a small number of bonds, such as the interval 2010-2020 for category AAA and 2004-2006 for AA, the standard errors become much smaller. This can be attributed to the combination of data sets, which improves the density of the distribution of maturity dates.

Since the total number of parameters in the MC model is smaller than in the corresponding SC models, the goodness of fit may be expected to decrease. This is also 'predicted' by the familiar trade-off between flexibility and smoothness. However, the price we have to pay for the improvements in smoothness and reliability is rather limited, because the RMSE statistics in table 4 are approximately equal for the SC and MC model for all rating categories. The scatter plots in figure $2 \mathrm{~b}$ also show about the same dispersion of the residuals and do not reveal any significant biases. Apparently, the focus on the credit spread and the joint estimation with the default-free government curve, offset the negative effects of the imposed parsimonious structure.

Finally, we provide a justification for the MC model specifications in table 3. Along the way, we illustrate that the favourable properties of the MC model cannot be obtained from using a parsimoniously specified corporate SC model or from a richly specified MC model. Moreover, we show that the shape of the spread curve in the SC model is sensitive to the precise specification of the model, while the MC model is relatively robust to model misspecification. We describe the specification procedure for A-rated bonds.

As starting point for the specification of the A discount spread curve in the MC model, we choose the specification of A's discount curve in the SC model, i.e. a cubic spline with knot scheme $\{3,9\}$. Subsequently, we reduce the flexibility of the spline model by lowering the degree or the number of knots (or both), because

\footnotetext{
class. Within a data set of bonds with the same rating, usually the longest maturity bonds have been issued by the relatively most credit worthy firms. Therefore, credit spreads may decrease for the longest maturities in such a data set.

${ }^{13}$ Note that we cannot judge the change in reliability of the spread curves, because we are unable to calculate standard errors of estimated spreads for the SC case. With SC models, the parameters of the government and corporate term structure models are estimated independently, so that we do not get the required covariances.
} 
we pursue a smooth, intuitively appealing spread curve. Including the initial combination, this yields 6 alternative specifications: degree 3 , knots $\{3,9\} ; 3,\{3\} ; 3,\{9\} ; 2,\{3,9\} ; 2,\{3\}$ and $2,\{9\}$. The specification for the government curve is unchanged as a cubic spline with knots $\{3,9\}$.

Figure 4 shows estimated spot spread curves from the MC model for the 6 combinations. For comparison, the figure also contains spot spread curves that are calculated from independently estimated SC models, where the specification of the GOVT discount curve is unchanged and the A discount curve is specified according to one of the 6 degree-knots combinations. The upper-left hand graph in figure 4 depicts the estimated SC and MC spreads for the first combination. The shape of the MC spread is just as twisty as that of the SC model. It is therefore clear that an MC model with the same number of parameters as an SC model, does not yield the described favourable results of a parsimoniously specified MC model. Figure 4 also reveals that the confidence bounds of the spot spread of a richly specified MC model are wider than that of the spot curve, cf. figure 2 .

\section{[insert figure 4 around here]}

We can smooth the spread curve by decreasing the spline degree and reducing the set of knots. We apply this idea for both the corporate discount curve in the SC model and the corporate discount spread curve in the MC model. The graphs in figure 4 reveal that the shapes of the SC spot spread curves calculated with these new settings vary considerably. This shows that estimating a parsimoniously specified SC model does not yield smooth and intuitively shaped spread curves. The spread curves obtained from the MC model, however, stiffen and are not as sensitive to different degree-knots settings. This robustness to model specification is a distinct advantage of the MC model over its SC competitor. The confidence bounds of the spot spread curve narrow

with the reduction of the number of parameters. Particularly a $2^{\text {nd }}$ degree spline model with one knot at 9 years has much tighter confidence bounds than the initial specification with a $3^{\text {rd }}$ degree spline with knots at 3 and 9 years. Since this model also yields a monotonically increasing spot spread curve, this is the preferred specification for the category of A-rated bonds.

Repeating the described procedure for the other bond categories yield remarkably similar conclusions: MC spreads are less sensitive to the specification of the discount spread model than the SC discount curve model; by lowering the number of parameters, confidence intervals tighten from their initial large width to more narrow bounds; a parsimoniously specified MC model is preferable due to an intuitively appealing shape and highest reliability. We find that a quadratic spline with one knot at 9 years is most convenient for categories 
$\mathrm{AAA}$ and $\mathrm{AA}$, and a quadratic spline with one knot at 5 years is preferable for $\mathrm{BBB}, \mathrm{BB}$ and $\mathrm{B}$.

\section{Conclusions}

We present a new framework for the joint estimation of term structures and credit spreads. By decomposing a corporate term structure in a default-free curve and a credit spread curve, we can use a parsimoniously specified model for the spread curve and take the default-free part from the government curve. Both the government and the spread model are modelled as $B$-splines and their parameters are jointly estimated from a combined data set.

We use a high-quality data set of German mark denominated bonds, with ratings ranging from AAA to B. For comparison, we first independently estimate separate single-curve term structure models for each of the rating classes. Spread curves that are obtained by subtracting the estimated government and corporate curves, have unrealistically twisting shapes and we argue that this can be attributed to the relatively small magnitude of spreads compared to interest rates. Therefore, any dissimilarity in the curvatures of the government and corporate curves implies twists in the spread curve. These twisting spread curves contradict the smooth curves that are predicted by the theoretical bond price models of Merton (1974), Longstaff and Schwartz (1995) and Jarrow et al. (1997).

Then we apply the proposed multi-curve model that explicitly and parsimoniously models the spread curve and jointly estimates it with the government curve. We illustrate that the model yields smooth spread curves that are more in line with the theoretical bond price models. Because the parameters are estimated from a combined data set of government and corporate bonds, the reliability of estimated term structures improves considerably. This effect is strongest for those maturity segments of a term structure that contain only a small number of bonds for that particular rating class. The ability of the multi-curve model to accurately fit market prices of bonds is hardly affected, in spite of the smaller number of parameters. We find that the favourable results of the model can attributed to both the joint and the parsimonious modelling of the spread curves.

The new term structure estimation framework is valuable for each model that requires accurately estimated term structures for different credit risk classes. Examples are pricing models for corporate bonds, models that value credit derivatives and models that asses credit risk in derivative products. 


\section{References}

Adams, K. J. and van Deventer, D. R. (1994), 'Fitting yield curves and forward rate curves with maximum smoothness', Journal of Fixed Income 4(1), 52-62.

Anderson, N., Breedon, F., Deacon, M., Derry, A. and Murphy, G. (1996), Estimating and interpreting the yield curve, John Whiley \& Sons, Ltd., Chicester.

Beim, D. O. (1992), Term structure and the non-cash value in bonds, Working Paper 92-40, Graduate School of Business, Columbia University.

Bliss, R. (1997), 'Testing term structure estimation methods', Advances in Futures and Options Research 9, 197-231.

Bühler, W., Uhrig-Homburg, M., Walter, U. and Weber, T. (1999), 'An empirical comparison of forward-- and spot-rate models for valuing interest-rate options', Journal of Finance 54(1), 269-305.

Helwege, J. and Turner, C. M. (1999), 'The slope of the credit yield curve for speculative-grade issuers', Journal of Finance $\mathbf{5 4}$ (forthcoming).

Jarrow, R. A., Lando, D. and Turnbull, S. M. (1997), 'A Markov model for the term structure of credit spreads', Review of Financial Studies 10(2), 481-523.

Langetieg, T. C. and Smoot, J. S. (1989), 'Estimation of the term structure of interest rates', Research in Financial Services 1, 181-222.

Litzenberger, R. H. and Rolfo, R. (1984), 'An international study of tax effects on government bonds', Journal of Finance 39(1), 1-22.

Longstaff, F. A. and Schwartz, E. S. (1995), 'A simple approach to valuing risky fixed and floating rate debt', Journal of Finance 50(3), 789-819.

McCulloch, J. H. (1971), 'Measuring the term structure of interest rates', Journal of Business 44, 19-31.

McCulloch, J. H. (1975), 'The tax-adjust yield curve', Journal of Finance 30(3), 811-830.

Merton, R. C. (1974), 'On the pricing of corporate debt: the risk structure of interest rates', Journal of Finance $\mathbf{2 9}(2), 449-469$. 
Poirier, D. J. (1976), The Econometrics of Structural Change, North Holland Publishing Company.

Powell, M. J. D. (1981), Approximation Theory and Methods, Cambridge University Press, Cambridge.

Shea, G. S. (1984), 'Pitfalls in smoothing interest rate term structure data: equilibrium models and spline approximations', Journal of Financial and Quantitative Analysis 19(3), 253-269.

Shea, G. S. (1985), 'Interest rate term structure estimation with exponential splines: A note', Journal of Finance 40(1), 319-325.

Steeley, J. M. (1991), 'Estimating the gilt-edged term structure: basis splines and confidence intervals', Journal of Business Finance $\mathcal{E} 3$ Accounting 18(4), 513-529.

Vasicek, O. A. and Fong, H. G. (1982), 'Term structure modeling using exponential splines', Journal of Finance 37(2), 339-348. 


\begin{tabular}{lccccccccc}
\hline \hline & GOVT & AAA & AA & A & BBB & BB & B & NR & Total \\
\hline All & 112 & 411 & 329 & 119 & 30 & 53 & 38 & 199 & 1291 \\
Included & 93 & 228 & 145 & 53 & 13 & 16 & 16 & 60 & 624 \\
\hline \hline
\end{tabular}

Table 1: Distribution of bonds in the data set by rating. All are all German Mark Eurobonds that are quoted at least once in June 1998. Included are fixed-income, bullet bonds that are quoted on at least 18 days of the 20 trading days of June 1998. Rating symbols: GOVT: AAA-rated government bonds; NR: not rated; AAA, AA, A, BBB, BB, B: Standard and Poor's major rating letters.

\begin{tabular}{lccccc}
\hline \hline & Financials & Government Agencies & Supra-Nationals & Industrials & Total \\
\hline AAA & 139 & 23 & 59 & 7 & 228 \\
AA & 99 & 33 & & 13 & 145 \\
\hline \hline
\end{tabular}

Table 2: Distribution of 'included' bonds in the data set by rating and industry. Financials consists of Reuters classifications 'Banks' and 'Financials'; Government Agencies is made up by 'Government National', 'Government Regional' and 'Government Agency'; Supra-National consists of 'Worldbank', 'InterAmerican Development Bank' and 'Supra-National'. Industrials is comprised of 'Gas \& Transmission', 'Utility \& Electricity', 'Transport-Nonrail', 'Telephone' and 'Industrial'.

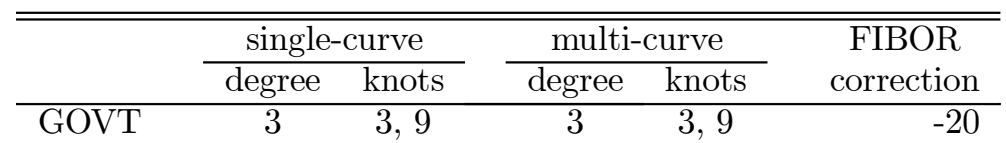

\begin{tabular}{llcllr} 
AAA & 3 & 3,9 & 2 & 9 & -10 \\
AA & 3 & 3,9 & 2 & 9 & 0 \\
A & 3 & 3,9 & 2 & 9 & +10 \\
BBB & 3 & 5 & 2 & 5 & +20 \\
BB & 3 & 5 & 2 & 5 & +30 \\
B & 3 & 5 & 2 & 5 & +40 \\
\hline \hline
\end{tabular}

Table 3: Model specifications for single-curve and multi-curve models. Degree and knots refer to the specification of the $B$-splines. FIBOR correction is the spread applied to Frankfurt Interbank Offered Rates (FIBORs) in calculating the prices of synthetic bonds to take credit risk into account. 


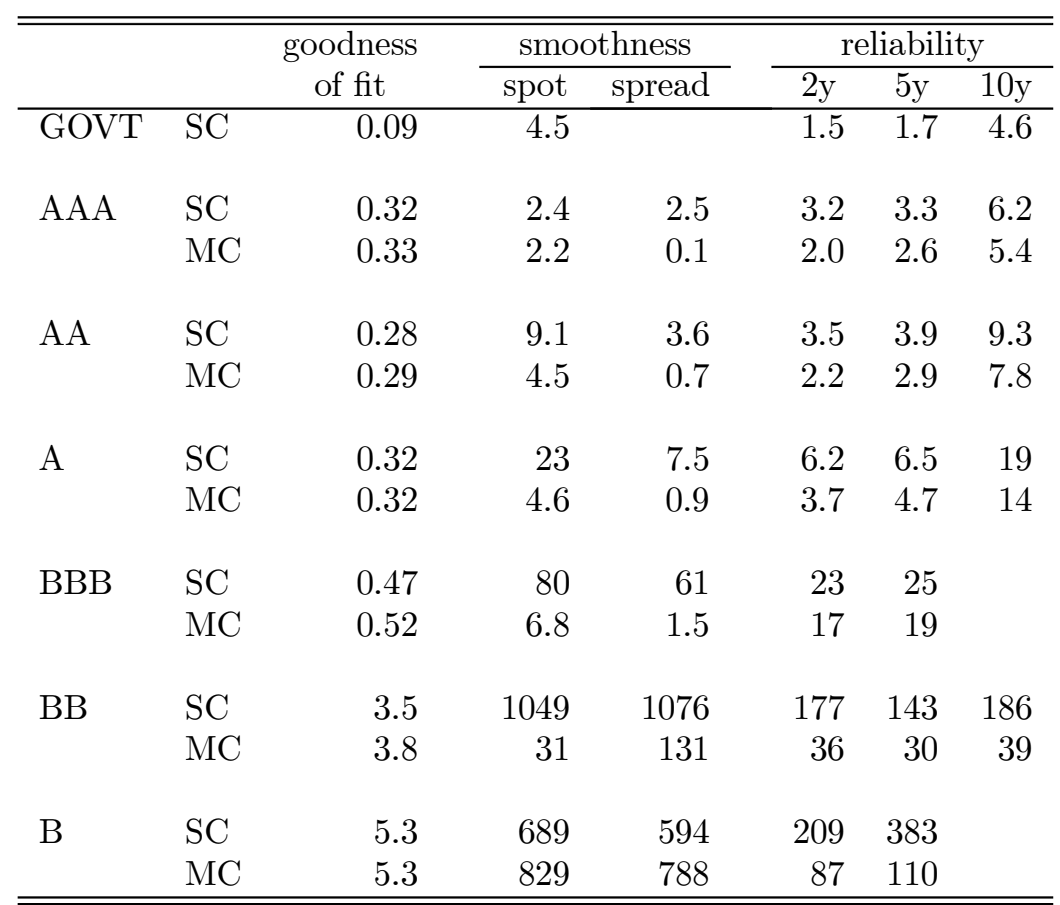

Table 4: Summary statistics of single-curve (SC) and multi-curve (MC) estimates, averaged over all 20 trading days of June 1998. Goodness of fit is calculated as the Root Mean Squared Error (RMSE) of the residuals. Smoothness is measured as $\left(10^{8}\right.$ times) the integral of the square of the second derivative, and is calculated for the spot curve and the spot spread curve. Reliability is ( $10^{4}$ times) the standard error of the estimated spot curve, evaluated at maturities 2,5 and 10 years.

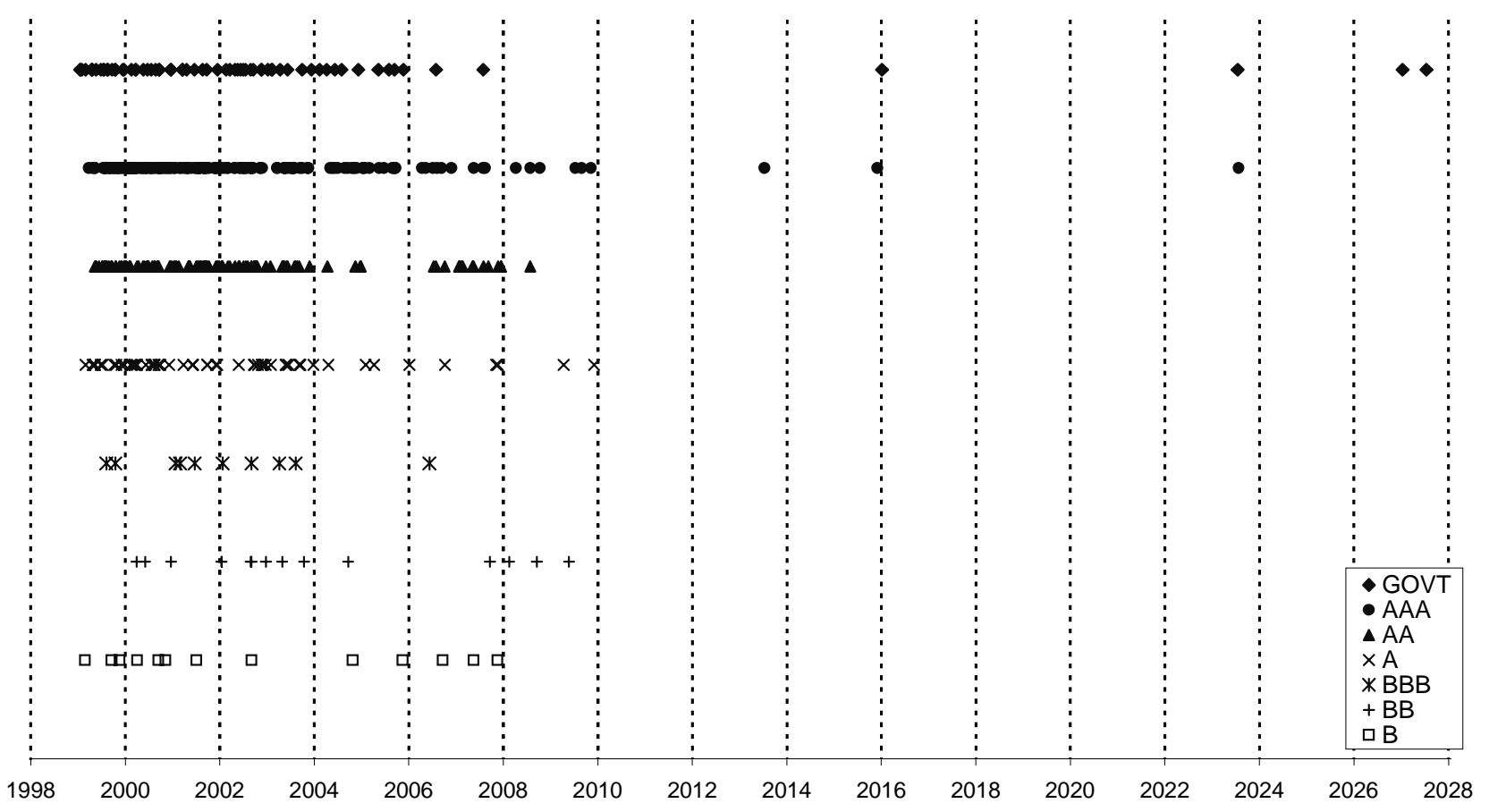

Figure 1: Distribution of the maturity dates of the bonds in the estimation sample by rating category. 

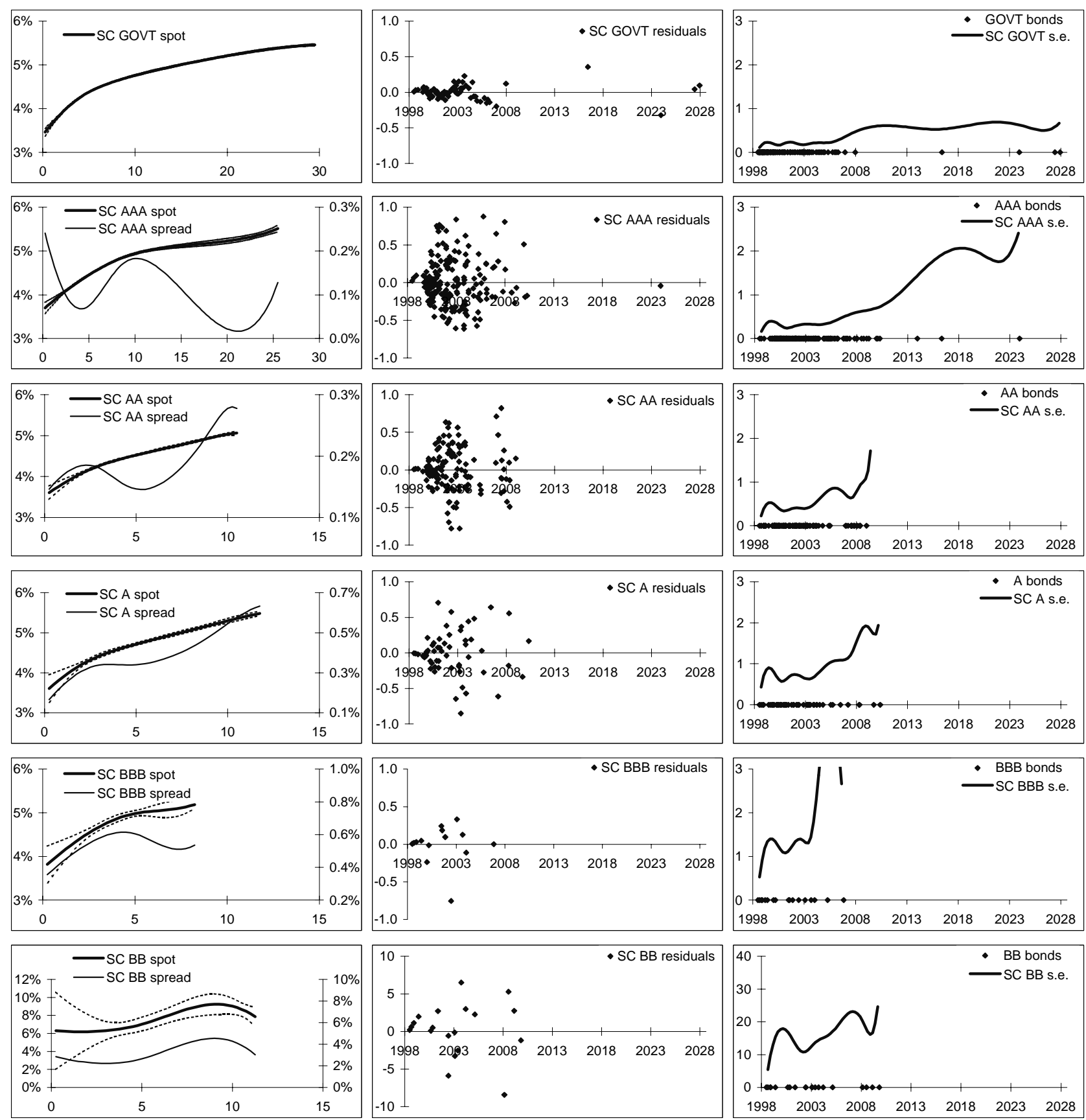

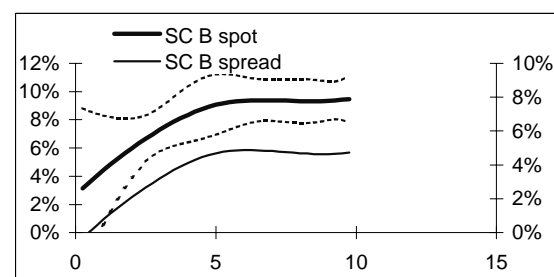

(a)

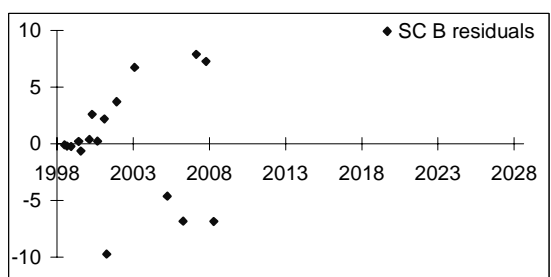

(b)
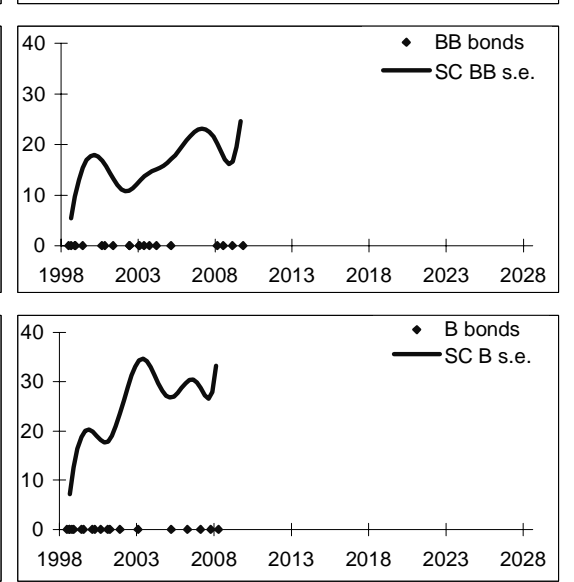

(c)

Figure 2: Single-curve (SC) estimates for June $2^{\text {nd }}$ 1998. Graphs (a) show estimated spot curves (with $95 \%$ confidence intervals) and spot spread curves; graphs (b) contain residuals; graphs (c) display (1000 times) the standard errors (s.e) of estimated discount curves. 

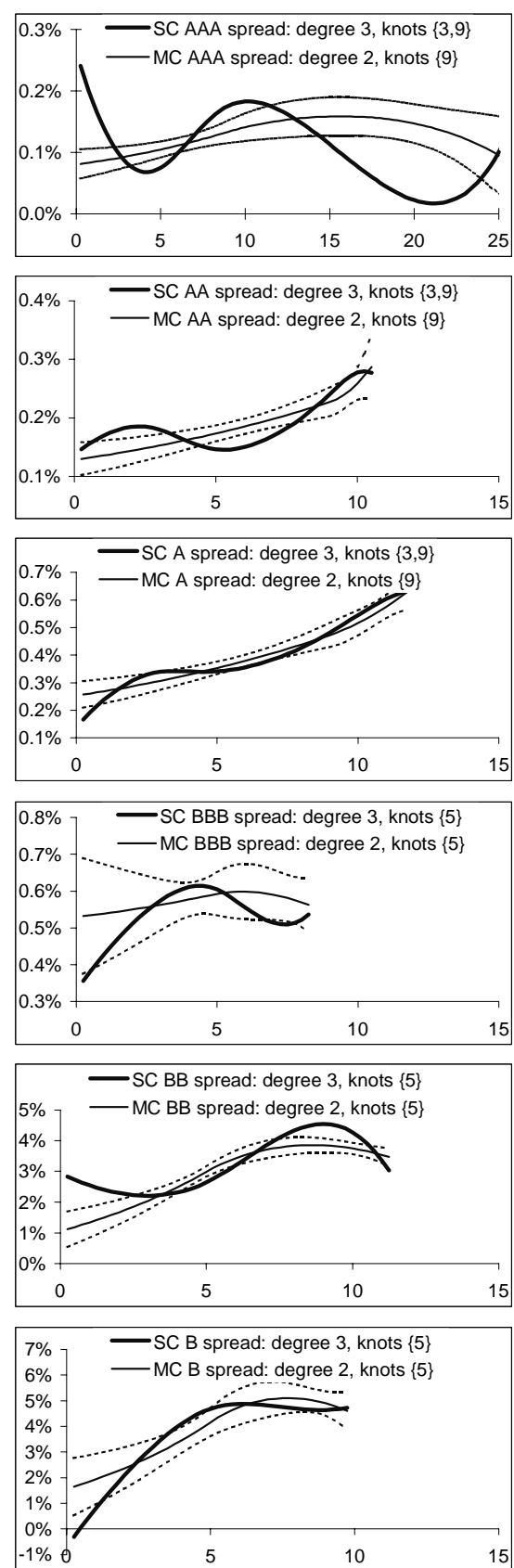

(a)
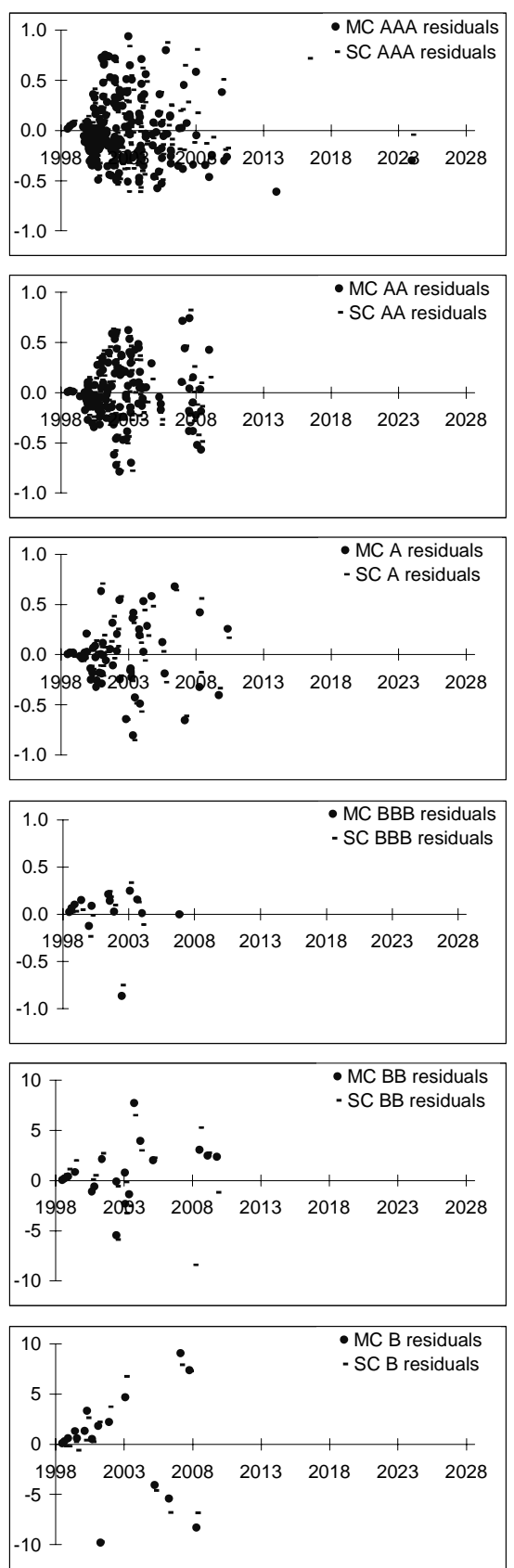

(b)
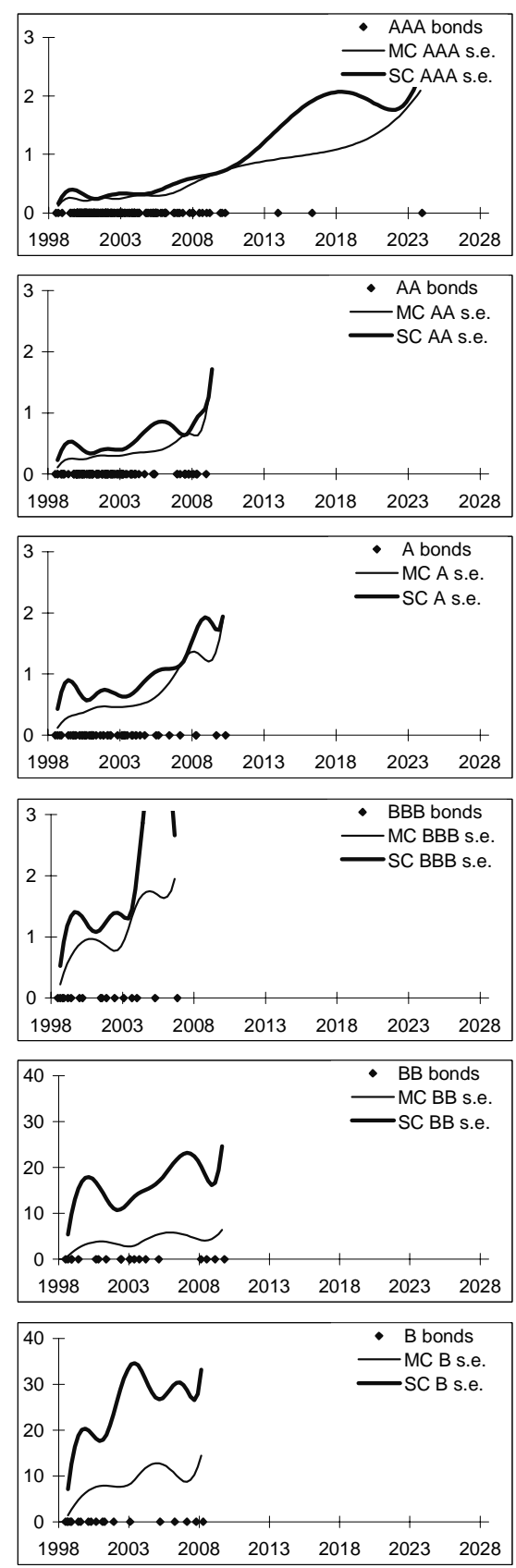

(c)

Figure 3: Single-curve (SC) and multi-curve (MC) estimates for June $2^{\text {nd }}$, 1998. Graphs (a) show spot spread curves (with $95 \%$ confidence intervals for MC spreads); graphs (b) contain residuals; graphs (c) display (1000 times) the standard errors (s.e) of estimated discount curves. 

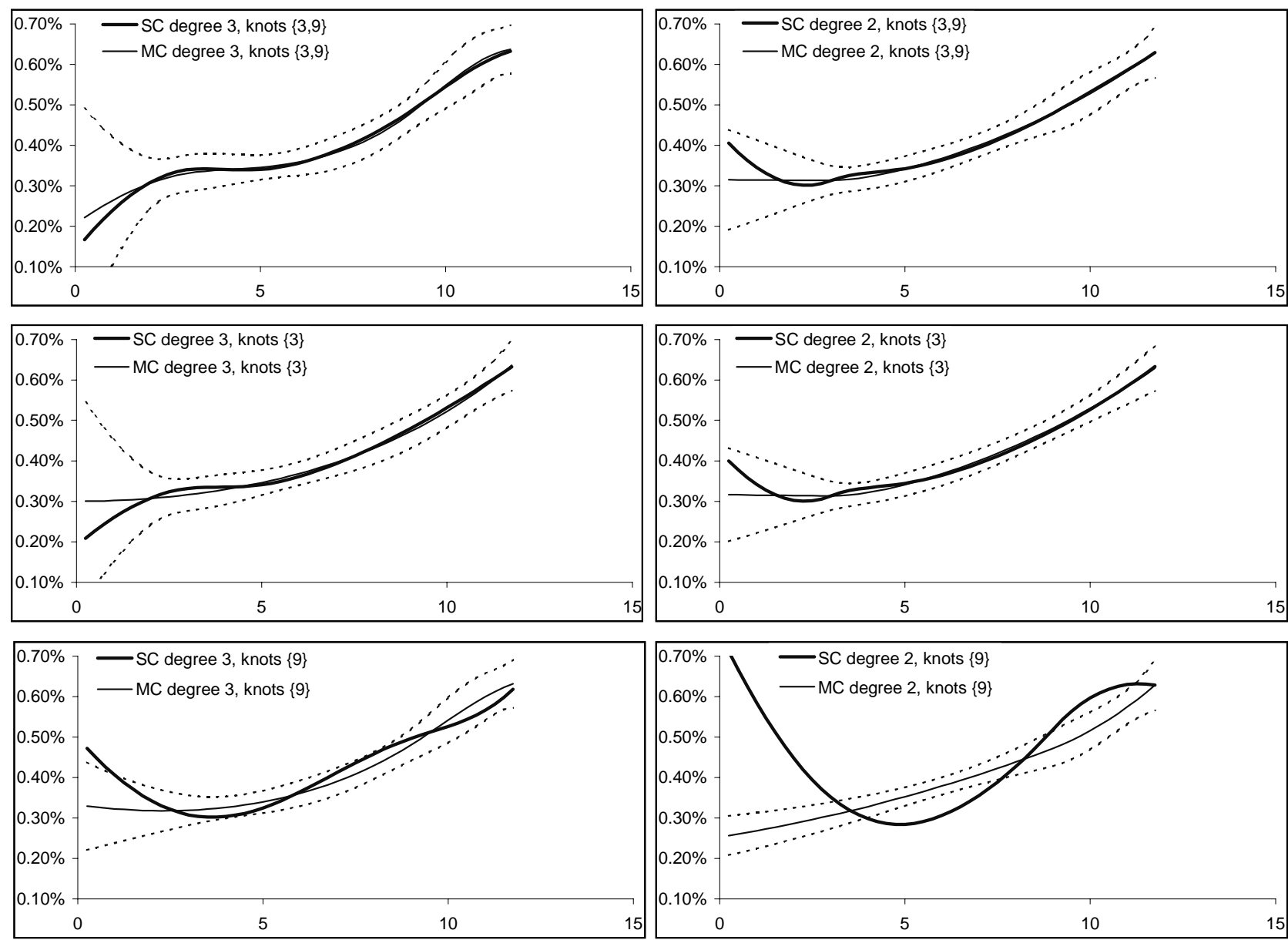

Figure 4: Single-curve (SC) and multi-curve (MC) spot spread curves for category A on June $2^{\text {nd }}$, 1998 obtained with different settings of the degree and the knots. 